\title{
Case Report of a True Accessory Mandibular Condyle - an Exceptionally Rare Abnormality
}

\author{
Ayesha Siddika $^{1}$, A M Ferdousi $^{2}$
}

\begin{abstract}
Mandibular accessory condyle is rare. Literature search found most of the accessory condyles to arise from the mandibular coronoid process due to hyperactivity of attached temporalis muscle. Although neoplastic growth at mandibular coronoid mimicking an accessory condyle also been cited. Absence of report in recent publications regarding accessory mandibular condyle arising from the main mandibular condyle makes this anomali extremely rare. The present case report is about a true accessory mandibular condyle which caused the patient facial and occlusal disharmony. A 3D computerized tomographic imaging ascertained the exact location of the accessory condyle, its origin and resting position of the accessory and the main condylar head. The facial and occlusal disharmony settled completely within a short postoperative time following surgical intervention and mild elastic traction.
\end{abstract}

Keywords: Accessory mandibular condyle; bifid condyle; radiological imaging.

Delta Med Col J. Jan 2016;4(1):45-50

\section{Introduction}

Accessory mandibular condyle is a rare condition. This anomaly very occasionally occurs at the coronoid process of mandible as hyperplasia due to over activity of temporalis muscle, chondroma and trauma. ${ }^{1}$ A true accessory mandibular condyle occurring at the neck of mandibular condyle is an extreme rare case. The literature search failed to find a reported case in recent publications. Although, bifid mandibular condyle was first reported by Hrdlicka $^{2}$ in 1941, which she evidenced in dried specimens of museum collection. Since then some cases of mandibular bifid condyle have been reported. Most cases of bifid condyles were symptomless and incidentally discovered on radiograph when investigating other problem. ${ }^{3}$ Nonetheless symptomatic bifid condyle with temporomandibular joint (TMJ) ankylosis has also been reported. ${ }^{4}$ Possibility exists for increased number of recent case reports are due to improved health awareness among population, recognition of problem by the subject and improved radiological imaging techniques have influenced the increase in number of case reports. ${ }^{5}$

Trauma has been mentioned as the most common cause of pathological change and abnormal/altered growth pattern in mandibublar coronoid/condylar area which mimicks an accessory mandibular condyle. 6,7

1. Assistant Professor (Hon.), Dept. of Maxillofacial surgery, Dental Unit, Delta Medical College, Dhaka, Bangladesh.

2. Professor, Dept. of Maxillofacial surgery, Dental Unit, Delta Medical College, Dhaka, Bangladesh.

Correspondence: Prof. A M. Ferdousi.e-mail: amfoms@yahoo.com 
The present article describes a true second condyle at the base of main condylar neck and the patient had no history of traumatic incidence.

\section{Case report}

A 38 year Asian female presented with a gradual change in her facial form having a gradual twist of chin towards her right side. There was no other associated symptom. Basically she was more concerned to her look. Clinically she had some deviation of her chin towards right in closed jaw position (Fig. 1). Intraoral examination evidenced a cross bite at her lower right lateral incisor to first premolar. Her jaw opening and lateral excursions were normal and the mandible did not deviate during opening of the jaw (Fig. 2), although on closure she landed in cross bite in right lateral incisor to first premolar region (Fig. 3). She also showed slightly increased over jet in the region of left canine and first premolar.

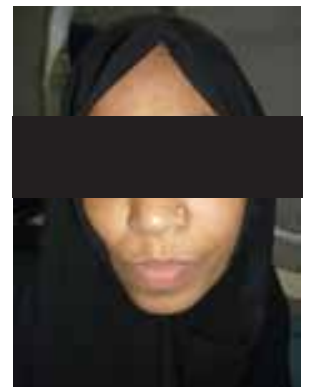

Fig. 1: Shift of chin to right

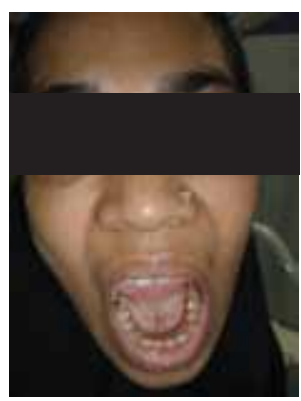

Fig. 2: No deviation of jaw on opening
Some degree of open bite was evidenced in the left sided occlusion with unsatisfactory cuspal contact showing mild lingual shift of lower dentition. Clinical impression suggested towards hyperplasia of the left mandibular condyle despite the absence of significant size discrepancy between both the condyles of mandible on palpation.

Radiological imaging [OPG and 3D $\mathrm{CT}]$ evidenced an accessory condyle at the left mandible arising from the medial aspect of the neck of main condyle (Fig. 4). The accessory condylar head resting antero-medially anterior to articular eminence preventing the main condyle to return to its normal resting position in the glenoid fossa. The imaging system [3D CT] confirmed the main condyle resting in the anterior compartment of glenoid fossa keeping the posterior compartment of the fossa unoccupied (Fig. 5). This resulted a mild shift in the position of the lower dentition towards lingual and cross bite at lower right canine-premolar area with mild deviation of chin towards right.

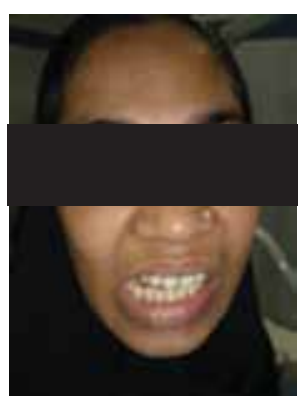

Fig. 3: Cross bite at right occlusion

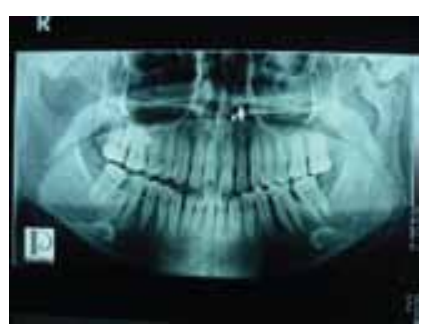

Fig. 4: Accessory left mandibular condyle
Patient was explained all about her condition and was advised surgical excision of the accessory condyle and was also warned about possible post-operative complications. She was mentioned about the possible need of elastic tractions postoperatively to keep the main left mandibular condyle in proper position in the glenoid fossa by gradual shifting of the soft tissue that has already filled the unoccupied space of posterior compartment of articular fossa. This may take some length of time to align the occlusion.

\section{Surgery}

Under general anaesthesia the patient was prepared for surgery with all aseptic measures and draping. Lignocaine $2 \%$ with 1:80000 adrenaline was injected at the left pre-auricular incision line subdermally and the incision access was made.

Following dissection, the left mandibular condylar capsule was reached and via an inverted L-shaped access through the capsule, the condyle was exposed. Careful dissection at the neck and medial 
aspect of the condyle, the accessory condyle was identified. Slightly opened and advanced position of mandible made the dissection and separation of soft tissue around the neck of accessory condyle easier. The accessory condyle was then excised from its base with rotary instrument and delivered out of the capsule (Fig. 6). By manipulation, the main condylar head was possible to bring back to its proper position in the articular fossa, align the occlusion at affected side and reduce the cross bite on contralateral side. It was evidenced, on withdrawal of manipulation the left condyle relapsed to its anterior position in the glenoid fossa. However, following complete haemostasis the capsular access was closed with 3.0 vicryl and the access wound was closed in layers with 3.0 vicryl and 5.0 skin sutures. The recovery was uneventful. She was released home in 48 hours and was advised mild jaw exercise.

At the follow up (7th postoperative day) patient's healing was satisfactory and sutures were removed. Her occlusion had no significant change but she was able to take her mandible posteriorly in occlusion with effort. Elastic rubber bands were placed on the affected (left) side around arch bars placed at upper and lower dental arches for mild elastic tractions to facilitate postero-lateral move on left side. She was advised 3 weeks follow up.

During the next follow up her swelling and pain at the left TMJ area improved satisfactorily. She was able to move her mandible freely with further reduction of her cross bite at contra lateral canine-premolar area along with improvement in deviation of chin to some extent. The left sided elastic tractions were reinforced by additional rubber elastics. She was then seen after one month when her cross bite resolved, chin position was almost normal and her occlusion settled to satisfaction with complete intercuspal contacts in both sides (Fig. 7). She was seen following further six weeks when the patient was doing very well. Her chin had settled in midline (Fig. 8), cross bite resolved and had good intercuspal articulation in both side of the jaws (Fig. 9) and had no complication at all.

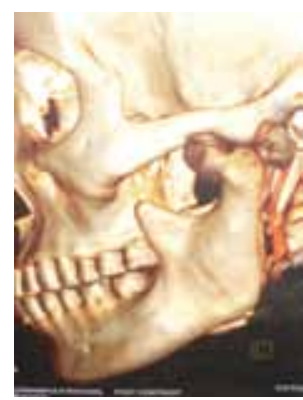

Fig. 5: Anteriorly resting position of left condyle

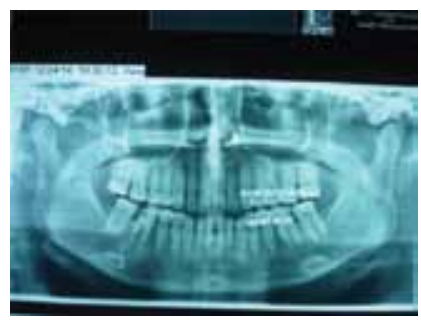

Fig. 7: Good intercuspal contact

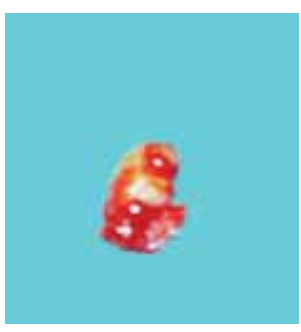

Fig. 6: Excised accessory condyle

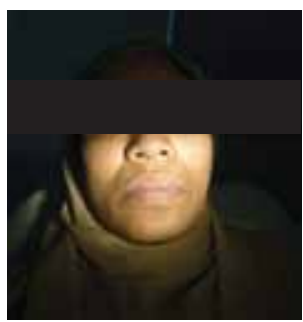

Fig. 8: Normal chin position

\section{Discussion}

Mandibular developmental abnormalities are not uncommon and often seen as part of a syndrome or even as a single entity. Macrognathia, micrognathia, bird face and mandibular condylar hyperplasia are often seen giving rise to facial deformity. Malocclusion, mandibular bowing, deviation of chin to one side are usual clinical presentation with or without TMJ symptoms in mandibular condylar abnormality.

Mandibular condylar hyperplasia alone can produce derangement in occlusion, bowing of mandible towards unaffected side, lateral cross bite at the unaffected side with deviation of chin and often there is no symptom or problem. TMJ symptom may not be obvious when only the condylar neck elongates and the condylar size and shape remains without change. Symptoms usually occur when condylar head becomes enlarged and functions become difficult producing pain and clicking with deviation of jaw on movement. Whereas TMJ pain dysfunction syndrome is mostly without change in a mandibular condyle, produces deviation of jaw on opening only due to muscular malfunction or spasm. 
Accessory mandibular condyle at coronoid process is rare. This anomaly is believed to be due to temporalis muscle hyperactivity, trauma and may occur due to a neoplasm. ${ }^{1}$ Accessory condyle may cause hypomobility of mandible due to obstruction in the movement within temporal fossa beneath the zygomatic arch. Accessory condylar contact at zygomatic arch and forming a pseudo joint "Jacobes disease", results growth hinderness and reduced jaw movements.

Malocclusion can be of dental or skeletal based and may also be compromised. Treatment for malocclusion deserves cephalometric assessment prior to treatment planning and may involve orthodontic, orthognathic surgery and even combined to obtain the best possible result.

Condylar hyperplasia (involving condylar neck) usually produces a mild bowing of mandible and chin towards the unaffected side and shows deviation of jaw from central line on opening of the jaw. Interestingly in this case there was no deviation of mandible from centre line on opening and the deviation of chin was only observed on closure of the jaw instead, which indicated incomplete journey of the left condyle to normal resting position due to possible mandibular condylar pathology. An accessory mandibular condyle was confirmed on radiograph. Suspicion may arise whether the abnormality could have been a bifid mandibular condyle which is commoner than our reported abnormality. ${ }^{8}$ Therefore the developmental anomaly such as bifid condyle remains a major possibility of presenting as an accessory condyle when the cleavage of bifid area extends inferiorly to condylar base. Possibility also remains of dual attachment of lateral pterygoid muscle in two sleeves at mandibular neck. When this occurs, hyperactivity of lateral pterygoid muscle produces a pull at both ends of the attached pterygoid muscle sleeves transferring the pulling force at different sites of condylar neck. This influences the condylar growth at both sites of muscle attachments as happens in accessory condyle at coronoid processes by over activity of temporalis muscles. ${ }^{9}$ Abnormal jaw growth and function develop along with the formation of jaw anomaly such as deviation of jaw from midline, malocclusion, open bites and disharmony in facial form.

A good number of cases of bifid condyle are in reports and recently the reported cases increased possibly due to improved techniques of imaging 10 (Fig. 10). The etiological pathogenesis of the bifid mandibular condyle has not been confirmed although various possibilities are mentioned. Dysplastic process during development in cartilage producing abnormal change ${ }^{11}$, trauma

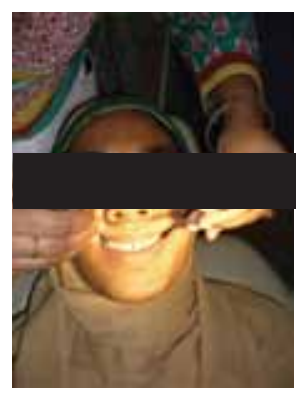

Fig. 9: Improved occlusion

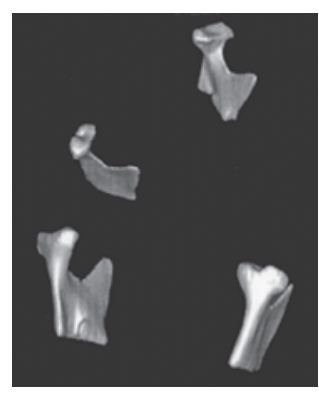

Fig. 10: Bifid condyle induced change of position of fibroblastic cells around the disc surface and their possible influence in bone remodeling could result in bifid condyle. ${ }^{12}$ Persistence of fibrovascular septae in the developing condylar head has been suggested as cause of the bifid condyle. Persistence of these septae at condylar head beyond second year of age could cause impaired ossification. ${ }^{13}$ A number of other factors - use of teratogenic substance during pregnancy, infection, irradiation, nutritional disorders, genetics, endocrinological or pharmacological factors also been pointed to cause bifid condyle in off springs of experimental rats. ${ }^{8}$ Trauma to the TMJ and even micro trauma due to malocclusion has been suggested to cause bifid condyle and ankylosis of TMJ. $3,5,14,15$

Diagnosis may become a dilemma in facial deformity to ascertain between hypoplasia, hyperplasia and abnormality. Even an improper radiological imaging may mislead an actual defect. Careful and detailed clinical assessment, proper 
radiological imaging and correct planning of treatment can make procedures and outcome satisfactory. Conventional imaging as panoramic view is common and important tool to diagnose condylar defect. Improved new techniques as computed tomography should be the choice for TMJ joint pathologies as this technique allows bilateral visualization without superimposition effect. ${ }^{10}$ Three dimensional CT [3D CT] reconstructed images enable the radiologist to visualize the condyle and its relationship with the base of skull and surrounding structures, as well as to manipulate the image data quickly. ${ }^{16}$

Mandibular condylar abnormality often remains asymptomatic when no treatment is prescribed. Even when an episode of symptom begins careful examination and investigation finds the underlying pathology and may improve with jaw exercise and medication. Only when facial or jaw asymmetry is evidenced and is unacceptable to the subject, surgical intervention becomes of absolute necessity. ${ }^{7}$

Facial deformity may occur from abnormal growth pattern of single facial skeletal bone or multiple bony abnormalities. Detailed clinical observation and proper radiological imaging are best way to reach clinical diagnosis and plan a treatment. In our case 3D CT view has been accurately helpful to ascertain the site of origin of the accessory mandibular condyle and plan a cautious surgery. Should the accessory condyle be originated from the coronoid process, as in most cases, intervention within the capsule was not necessary. In the present case the site of origin of the abnormality being at the main condylar neck surgical access had to be through the capsule and precaution against the damage to the associated anatomical structures such as maxillary artery, Vn and VIIn branches had to be kept in mind. Postoperatively, any necessity of additional treatment depends on regular follow up and observation.

\section{References}

1. Peacock ZS, Resnick CM, Faquin WC, Kaban LB. Accessory Mandibular Condyle at the Coronoid Process. The Journal of Craniofacial Surgery. 2011;22(6):2168-71.

2. Hrdlička A. Lower Jaw: Double Condyles. American Journal of Physical Anthropology. 1941;28(1):75-89.

3. Artvinli LB, Kansu O. Trifid Mandibular CondyleA Case Report. Oral Surgery Oral Medicine Oral Pathology Oral Radiology. 2003;95(2):251-54

4. Sales MAO, Oliveira JX, Cavalcanti MGP. Computed Tomography Imaging Findings of Simultaneous Bifid Mandibular Condyle and Temporomandibular Joint Ankylosis: Case Report. Braz Dental J. 2007;18(I):74-77.

5. Heserk N, Ozbek M, Tasar F, Akpinar E, Firat M. Bifid Mandibular Condyle: A Case Report. Dental Traumatology. 2004;20(3):184-86.

6. de Mello LS, Barbosa JMN, Peixoto AC, Santana TS, Gerbi M. Bilateral Bifid Mandibular Condyle: A Case Report. Int J Morphol. 2011;29(3):922-26.

7. Thomason JM, Yusuf H. Traumatically Induced Bifid Mandibular Condyle: Report of Two Cases. British Dental Journal. 1986;161(8):291-93.

8. Gunlach KK, Furhmann A, Beckmann-Van der Ven G. The Double-headed Mandibular Condyle. Oral Surgery Oral Medicine Oral Pathology Oral Radiology. 1987;64(2):249-53.

9. Szentpetery A, Kocsis G, Marcsik A. The Problem of the Bifid Mandibular Condyle. J Oral Maxillofac Surg. 1990;48(12):1254-57.

10. Tuncbilek G, Cavdar G, Mavili ME. Bifid Mandibular Condyle: A Rare Disorder. The Journal of Craniofacial Surgery. 2006;17(6):1207-209.

11. Martin GC, Teran TG, Reija MFG, Santolino SS, Bustillo RS. Bifid Condyle: Case Report. Medicina Oral Patologia Oral Y Cirugia Bucal. 2005;10(3):277-79.

12. Poswillo DE. The Late Effects of Mandibular Condylectomy. Oral Surgery Oral Medicine Oral Pathology Oral Radiology. 1972;33(4):500-12. 
13. Blackwood HJJ. The Double-headed Mandibular Condyle. American Journal of Physical Anthropology. 1957;15(I):1-8.

14. Fuentes FR, Flores HF, Mella HS, Lillo TF. Bifid Condylar Process: Case Report. Int. J. Morphol. 2009;27(2):539-41.
15. Standnicki G. Congenital Double Condyle of the Mandible - Causing Temporo mandibular Joint Ankylosis - Case Report. J Oral Surg. 1971;29(3):208-11.

16. Stefanou EP, Fanourakis IG, Vlastos K, Katerelou J. Bilateral Bifid Mandibular Condyles. Report of Four Cases. Dentomaxillofacial Radiology. 1998;27(3):186-88. 